\title{
Open Channels for Functional Bowel Disorders: Guanylate Cyclase C Agonists in IBS and CC
}

\author{
Eamonn M. M. Quigley
}

Published online: 29 June 2013

(c) Springer Science+Business Media New York 2013

Despite the high global prevalence and impact on the individual and society of irritable bowel syndrome and chronic idiopathic (functional) constipation (commonly referred to as chronic constipation or CC), new and effective therapies are remarkable by their paucity. Several factors have conspired to restrict the physician's armamentarium in relation to functional bowel disorders, including the intrinsic heterogeneity of these entities, an absence of a unifying explanation for their pathophysiology, and a very low tolerance among regulatory agencies for any adverse events in the treatment of disorders so commonly, and inappropriately, regarded as nothing more than a nuisance. As a consequence, optimal therapeutic targets remain difficult to define, ideal outcome measures the subject of much debate, and the selection of the most appropriate study population a process that owes as much to art as science. Agents directed at the traditional targets, motility and visceral sensation, have in the past, been troubled, not only by marginal efficacy, but also by a prevalence of adverse events deemed unacceptable [1]. More recent refinements, such as the development of highly selective receptor agonists, seem to have overcome some of these hurdles, as exemplified by studies with the selective $5-\mathrm{HT}_{4}$ agonist, prucalopride, in chronic constipation [2]. Indeed, this drug has now been approved for the treatment of constipation in several jurisdictions.

Spurred on, perhaps, by the specter of adverse events associated with systemically-acting compounds, others have taken a different route and sought to develop compounds that acted locally on the intestinal epithelium. The

E. M. M. Quigley ( $₫)$

Division of Gastroenterology, The Methodist Hospital, 6550 Fannin St, SM 1001, Houston, TX 77030, USA

e-mail: equigley@tmhs.org concept of a luminally-active or locally-acting agent in constipation is, of course, not new; these are the mechanisms of action of some of our most time-honored laxatives.

What is scientifically most appealing about the new batch of compounds is their foundation in epithelial biology. Each of these, lubiprostone, linaclotide and plecanatide, was primarily developed to promote intestinal chloride $\left(\mathrm{Cl}^{-}\right)$secretion which, by increasing luminal volume, facilitates stool passage. This goal is achieved through different physiological processes and, in some instances, is supplemented by other potentially beneficial effects. The first of these compounds to reach the clinic, lubiprostone, activates chloride secretion through effects on the apical chloride channel CLCN2 and the cystic fibrosis transmembrane regulator (CFTR), while the two newer compounds, linaclotide and plecanatide, are guanylate cyclase C (GC-C) peptide ligands.

Lubiprostone is a bicyclic fatty acid derived from prostaglandin E1 that was initially thought to exert its effects through activation of the apical chloride channel CLCN2 but is now considered to act primarily by activating the enterocyte apical anion channel CFTR through interaction with basolateral prostaglandin E4 (EP4) receptors. [3]. Linaclotide, a 14-amino acid peptide, and plecanatide (structurally highly similar to the physiological agonists of GC-C receptors, uroguanylin and guanylin) bind to and activate guanylate cyclase $\mathrm{C}$ on the luminal surface of the intestinal epithelium. Activation of GC-C generates cyclic guanosine monophosphate (cGMP). An increase in intracellular cGMP within intestinal epithelial cells activates CFTR, increasing the secretion of $\mathrm{Cl}^{-}$and $\mathrm{HCO}_{3}{ }^{-}$into the lumen, increasing the rate of plasmalumen water flux thereby increasing stool volume [3-5] in a mechanism identical to the diarrhea associated with 
enterotoxigenic $E$. coli infection [5] although greatly attenuated.

In contrast to lubiprostone [6], linaclotide may stimulate motor activity [7]; lubiprostone, but not linaclotide, augments epithelial barrier resistance [8]. In IBS-C, linaclotide but not lubiprostone affects abdominal pain apparently independently of stool frequency [9], perhaps due to the effects of cGMP on visceral sensory afferents [10, 11]. The combination of an acceleration of colonic transit with its visceral analgesic effect and increased stool volume bodes well for the clinical impact of linaclotide and related compounds in IBS-C.

The recent paper by Shailubhai and colleagues in this journal now provides us with the first human data on the newest agent, plecanatide [12]. In this first-in-man study, plecanatide was administered over a very broad dose range to healthy volunteers. Using two assays, plecanatide was immeasurable in serum even with the very highest doses, confirming that, like lubiprostone and linaclotide, plecanatide is minimally, if at all absorbed. It was also well tolerated with diarrhea being its main adverse effect; however, the incidence of diarrhea was similar between plecanatide and placebo with few data supporting a doserelated effect. Nausea, which has been a relatively common adverse event with lubiprostone, occurred at the highest doses with an overall incidence of $5.7 \%$; abdominal pain and discomfort were also an issue at very high doses. Though the primary goal of this study was safety, some objective measures of gastrointestinal function were assessed. Nevertheless, no consistent effect on time to first bowel movement, Bristol stool form scale score, or stool frequency was reported [12]. Since the study subjects were normal volunteers, it is a little surprising that no effects on these measures of bowel function were observed, even at the higher doses; in its phase I study, linaclotide shortened the time to first bowel movement, decreased stool consistency, and increased stool frequency [5]. At the end of the day, however, outcomes of phase II and III clinical trials conducted in subjects diagnosed with $\mathrm{CC}$ and C-IBS will greatly inform the clinical utility of this compound. In this regard, in the preliminary results of a phase IIa study with plecanatide, impressive and beneficial improvement of time to first bowel movement, change from baseline in spontaneous and complete spontaneous bowel movements, and Bristol stool form score were reported, in addition to marked improvement in symptoms such as abdominal discomfort and straining and in global assessments [13]. A much narrower dose range (from 0.3 to $9 \mathrm{mg}$ ) was evaluated in this study, with beneficial effects evident at the lowest doses, plateauing at the $1 \mathrm{mg}$ dose; results of the phase IIb/III study which assessed the 0.3, 1 and $3 \mathrm{mg}$ doses will thus be of great interest.
These new agents, which offer much promise in the management of $\mathrm{CC}$ and IBS-C, raise some interesting scientific and clinical questions. The clinician will want to know when to use these agents in the management of these disorders; the answer is a little easier in IBS-C where there are relatively few alternatives but more complex in $\mathrm{CC}$ where competition is present with several time-honored laxatives and the potential arrival of a new generation of prokinetic agents. Ultimately, the prescriber will seek information on the relative efficacy and tolerability of these drugs in relation to each other and to standard therapy. On the scientific front, there are an equal number of questions. For example, what is the primary site of action of these agents, small intestine or colon? The pH-dependent action of plecanatide, akin to the physiological agonist urogunaylin but unlike linaclotide, suggests a more proximal small intestinal location for this agent [12], as would the distribution of cyclic GMP-dependent protein kinases, important mediators of the effects of GC-C agonists [5]. What relevance do these intrinsic properties have to drug tolerance and efficacy? Why do some of these agents cause nausea? What are the clinical implications of laboratory observations on the impact of lubiprostone on intestinal mucin and the gut microbiota? While the drugs discussed here have been deliberately designed to limit their actions to the intestine by greatly reducing or eliminating systemic absorption, compounds that increase the rate of anion secretion have the potential to impact a variety of diseases through effects on anion channels expressed in other epithelia and in the nervous system, sensory organs, skeletal muscle, bone and kidney [14]. Furthermore, activation of GC-C signaling also exerts anti-inflammatory and anti-neoplastic effects in models of ulcerative colitis and colon cancer, respectively [5]. Indeed, two mutations in the gene encoding GC-C-one, a gain-in-function mutation associated with a chronic diarrhea phenotype and the other, a loss-of-function mutation linked to a meconium ileus phenotype-underscore the biological relevance of this receptor and the pathways that it interacts with [5].

After several years of false dawns and much frustration for patients and their physicians the advent of new compounds like plecanatide does offer cause for some optimism in the management of some of medicine's most common, but challenging, disorders. Efficacy in $\mathrm{CC}$ and IBS-C has already been demonstrated for lubiprostone and linaclotide; results of phase IIb and III studies with plecanatide in these disorders are eagerly awaited.

\section{References}

1. Craig OF, Quigley EMM. Current and emerging therapies for the management of functional gastrointestinal disorders. Ther $A d v$ Chronic Dis. 2011;2:87-99. 
2. Camilleri M, Deiteren A. Prucalopride for constipation. Expert Opin Pharmacother. 2010;11:451-461.

3. Verkman AS, Galietta LJV. Chloride channels a drug targets. Nat Rev Drug Discov. 2009;8:153-171.

4. Lin JE, Valentino M, Marszalowicz G, et al. Bacterial heat-stable enterotoxins: translation of pathogenic peptides into novel targeted diagnostics and therapeutics. Toxins. 2010;2:2028-2054.

5. Brierly SM. Guanylate cyclase-C receptor activation: unexpected biology. Curr Opin Pharmacol. 2012;12:632-640.

6. Sweetser S, Busciglio IA, Camilleri M, et al. Effect of a chloride channel activator, lubiprostone, on colonic sensory and motor functions in healthy subjects. Am J Physiol Gastrointest Liver Physiol. 2009;296:G295-G301.

7. Andresen V, Camilleri M, Busciglio IA, et al. Effect of 5 days linaclotide on transit and bowel function in females with constipation-predominant irritable bowel syndrome. Gastroenterology. 2007;133:761-768.

8. Cuppoletti J, Blikslager AT, Chakrabarti J, Nighot PK, Malinowska DH. Contrasting effects of linaclotide and lubiprostone on restitution of epithelial barrier properties and cellular homeostasis after exposure to cold stressors. BMC Pharmacol. 2102;12:3.

9. Johnston JM, Kurtz CB, Macdougall JE, et al. Linaclotide improves abdominal pain and bowel habits in a phase IIb study of patients with irritable bowel syndrome with constipation. Gastroenterology. 2010;139:1877-1886.
10. Eutamene H, Bradesi S, Larauche M, et al. Guanylate cyclase C-mediated antinociceptive effects of linaclotide in rodent models of visceral pain. Neurogastroenterol Motil. 2010;22:312-e84.

11. Whitehead WE, Palsson OS, Gangarosa L, Turner M, Tucker J. Lubiprostone does not influence visceral pain thresholds in patients with irritable bowel syndrome. Neurogastroenterol Motil. 2011;23:944-e400.

12. Shailubhai K, Comiskey S, Foss JA, et al. Plecanatide, an oral guanylate cyclase $\mathrm{C}$ agonist acting locally within the gastrointestinal tract, is safe and well-tolerated in single doses. Dig Dis Sci. (Epub ahead of print). doi:10.1007/s10620-013-2684-z.

13. Shailubhai K, Talluto C, Comiskey S, Foss J, Joslyn A, Jacob G. A phase IIa randomized, double-blind, placebo-controlled, 14-day repeat, oral, dose-ranging study to assess the safety, pharmacokinetic and pharmacodynamic effects of plecanatide (SP- 304) in patients with chronic idiopathic constipation (Protocol No. SP-SP304201-09). http://www.sec.gov/Archives/edgar/data/1347613/00011046591005 2588/a10-19351_3ex99d1.htm. Accessed May 9, 2013.

14. Jentsch TJ, Maritzen T, Zdebik AA. Chloride channel diseases resulting from impaired transepithelial transport or vesicular function. J Clin Invest. 2005;115:2039-2046. 